\title{
Urodynamic assessment of short-term effects of pelvic radiotherapy on bladder function in patients with gynecologic cancers
}

\author{
Volkan Emirdar ${ }^{1}$, Umit Nayki ${ }^{2}$, Ibrahim E. Ertas ${ }^{1}$, Cenk Nayki ${ }^{2}$, \\ Mehmet Kulhan'ㄹ, Yusuf Yildirim² \\ ${ }^{1}$ Department of Gynecology and Obstetrics, Tepecik Education and Research Hospital, Izmir, Turkey \\ ${ }^{2}$ Department of Obstetrics and Gynecology, School of Medicine, Erzincan University, Erzincan, Turkey
}

\begin{abstract}
Objectives: To determine the short-term effects of adjuvant or primary curative radiotherapy (RT) on the urinary system in women with gynecologic cancer.

Material and methods: This is a prospective, concurrent cohort study including 55 patients with gynecologic cancer who were divided into three groups. Group 1 included 10 patients who were administered adjuvant RT following a radical hysterectomy (RH); Group 2 included 36 patients who were administered adjuvant RT following a type 1 hysterectomy and Group 3 included 9 patients who were administered primary curative RT. Urogynecologic assessments were carried out on patients before and six months after the treatment.

Results: Compared to pretreatment, no significant differences were observed in any of the three groups after treatment in terms of incontinence, first urge to urinate, normal urge to urinate, severe urge to urinate and changes in residual urine volumes. There was a significant decrease in maximal vesical pressure after treatment in Group 1 and Group 3. The maximum detrusor pressure decreased significantly in Group 1. The post-treatment decline in bladder capacity in Group 1 and Group 2 was also significant.

Conclusions: $\mathrm{RH}$ and pelvic RT cause lower urinary system dysfunction. Especially patients who receive primary curative $\mathrm{RT}$ and patients who are administered RT after $\mathrm{RH}$, where more pelvic denervation occurs, are at higher risk due to high doses of RT.
\end{abstract}

Key words: urinary incontinence, urodynamics, radiotherapy in gynecologic cancers

Ginekologia Polska 2016; 87, 8: 552-558

\section{INTRODUCTION}

Lower urinary system dysfunctions are frequently observed in benign and malignant gynecologic diseases due to close anatomical, embryological and functional relationships of the urinary and genital system. Symptoms emerge due to invasion or involvement of the urinary system, compression of the mass, or treatment of a pathologic condition [1]. Although urinary dysfunctions may develop secondary to malignancy, they frequently occur due to surgery and/or radiation therapy (RT) for the treatment of gynecologic cancer [2-4]. Urinary incontinence (UI) is the most important lower urinary system dysfunction caused by gynecologic cancer treatments. Other long-term morbidities include genitourinary fistula, ureteral stenosis, and hemorrhagic cystitis [5]. Symptoms originating from the lower urinary system bring out serious hygiene and social problems, and may result in the development of psychological symptoms such as insecurity, shame, guilt, and denial in the affected women [6]. They may also cause avoidance of social activities, isolation, and sexual function disorders, consequently leading to decreased quality of life (QoL) [7].

Nowadays, it seems crucial to keep QoL unaffected while increasing the lifespan as a result of advanced gynecologic cancer treatments. Data regarding pre- and post-treatment 
urodynamic evaluation of gynecologic cancer treatment methods and lower urinary system dysfunctions are limited. The aim of our study was to identify short-term (six months) effects of adjuvant or primary curative RT on the urinary system in women with gynecologic cancer, and to compare these with the results of urodynamic investigations before and after treatment.

\section{MATERIAL AND METHODS}

Fifty-five patients with endometrial cancer $(n=36)$, early-stage cervical cancer $(n=10)$, and local advanced cervical cancer $(n=9)$, who underwent adjuvant or primary RT at the Gynecologic Oncology Clinic of Tepecik Training and Research Hospital between 2011 and 2012, were included. Local Ethics Committee approved of the study and written informed consent was obtained.

Our study population was subdivided into three groups: 10 early-stage cervical cancer patients who received adjuvant RT due to lymphovascular space invasion (LVSI) in their type III radical hysterectomy $(\mathrm{RH})$ specimen (group 1); 36 endometrial cancer patients who received adjuvant RT after total abdominal hysterectomy and bilateral salpingo-oophorectomy (TAH + BSO), pelvic + para-aortic lymph node dissection and omentectomy (group 2); 9 patients with stage IIB or advanced stage cervical cancer who received directly primary curative RT (group 3). Patients who received systemic chemotherapy during curative RT were listed separately. Total pelvic dose of external radiotherapy (ERT), and total reference dose of intracavitary brachytherapy were recorded for each individual. Acute side effects of radiation which occurred either during RT procedure or within six months after radiation were considered. Urogynecologic assessment was performed twice: before treatment and six months after the treatment.

Detailed obstetric, gynecologic, and urologic history was obtained from all patients. The presence of UI was checked with different stress tests such as Valsalva and cough tests, which were performed at maximal bladder capacity. Subjects with UI were considered as "stress test" positive. Then, patients were asked to empty the bladder and post-voiding residue (PVR) volume was measured by inserting a sterile catheter through the urethral meatus. Uterus- or ovary-based pathologies were evaluated by transvaginal ultrasound and recorded. The ultrasound examination was repeated for post-treatment follow-up to detect signs of any surgical complications such as hematoma, lymphocyst or relapses. Urethral mobility was assessed by placing a swab through urethral meatus into the urethra under sterile conditions and measuring the angle between the swab and the horizontal plane during resting and straining. If the difference between the angles was more than $30^{\circ}$, urethral mobility was considered positive $[8,9]$. All patients were scanned with urinalysis before urodynamic assessment. Urinary cultures were procured when necessary. In the presence of any urinary tract infections, the urodynamic examination was postponed until the infection was cured.

Urodynamic assessment was performed with a "basic system urodynamics device" (Aymed Dyno, Urodynamics, Istanbul, Turkey). At the lithotomy position, a sterile 7F 3-lumen cystometry catheter was placed into the urethra and a rectal catheter with a $5 \mathrm{cc}$ balloon into the rectum to measure the abdominal pressure concurrently. The device was calibrated before each operation. The bladder was filled with physiological saline at room temperature at thespeed of $50 \mathrm{~mL} / \mathrm{min}$. During filling cystometry, bladder capacity and compliance, vesical pressures, and abdominal pressures were measured. Detrusor pressure was calculated automatically with the aid of a computer by subtracting the abdominal pressure from the vesical pressure (Pdet $=$ Pves - Pabd). At the time of bladder filling, the patients were questioned about their first urge to urinate, normal urge to urinate, and severe urge to urinate, and their vesical and detrusor pressures were recorded during that time.

The point which the patient declared a severe urge to urinate and could no longer delay urination was considered as "maximum cystometric bladder capacity". The upwards fluctuations in detrusor pressure due to spontaneous or provocative maneuvers such as coughing or straining were termed as "involuntary detrusor contractions". Whether or not the involuntary contractions resulted in incontinence, they were considered as an urodynamic indicator of an overactive detrusor. The pattern of detrusor contractions was noted as a single wave during bladder emptying. The patient was asked to cough at every $100 \mathrm{~mL}$ during bladder filling. Incontinence while coughing or thereafter was recorded. The increased abdominal pressure which leads to incontinence was termed as "abdominal leak point". "Urodynamic stress incontinence" was described as involuntary incontinence when abdominal pressure increased without any detrusor contraction and "detrusor instability" as incontinence occurring due to involuntary detrusor contractions.

Analyses were carried out using the SPSS 20.0 program (SPSS, Inc., Chicago, IL). Results were expressed as percentage and/or mean \pm standard deviation (SD). ANOVA and Kruskal-Wallis tests were used to measure differences of variables among the groups. Student's t-test was used for analyzing the differences of values before and after treatment within the groups. The tests were performed using the $95 \%$ confidence interval and the statistical significance was set at $\mathrm{p}<0.05$. 


\section{RESULTS}

There were no statistically significant differences between the three groups in terms of demographic, reproductive, and comorbidity-related characteristics except for age, the number of menopausal patients, and smoking rate. Mean age, number of menopausal patients, and smoking rate were significantly higher in Group 2 (Table 1). Additionally, radiation techniques and doses were shown in Table 2.

Pretreatment incontinence rates were similar in each group. However, a significant decrease in incontinence rate after treatment was only observed in Group 2 ( $p<0.05$ ). Similarly, pretreatment positive urethral mobility rate was significantly higher in Group $2(p<0.05)$ (Table 3).
Cystometry results of the groups were shown in Table 4. There was a significant decrease in the first urge to urinate volumes before and after treatment only in Group 2. On the other hand, the decrease in normal urge to urinate volumes before and after treatment was significant in both, Groups 2 and $3(p<0.05)$. For the severe urge to urinate volumes before and after treatment, significant decreases were observed in all of the investigated groups.

A significant decrease was found in post-treatment maximum vesical pressure in Groups 1 and 3 ( $p=0.012$ and $p=0.008$ ). In addition, there was a significant decrease in the maximum detrussor pressure before and after treatment only in Group 1 ( $p=0.009)$. On the other hand, significant

Table 1. Characteristics of the study groups

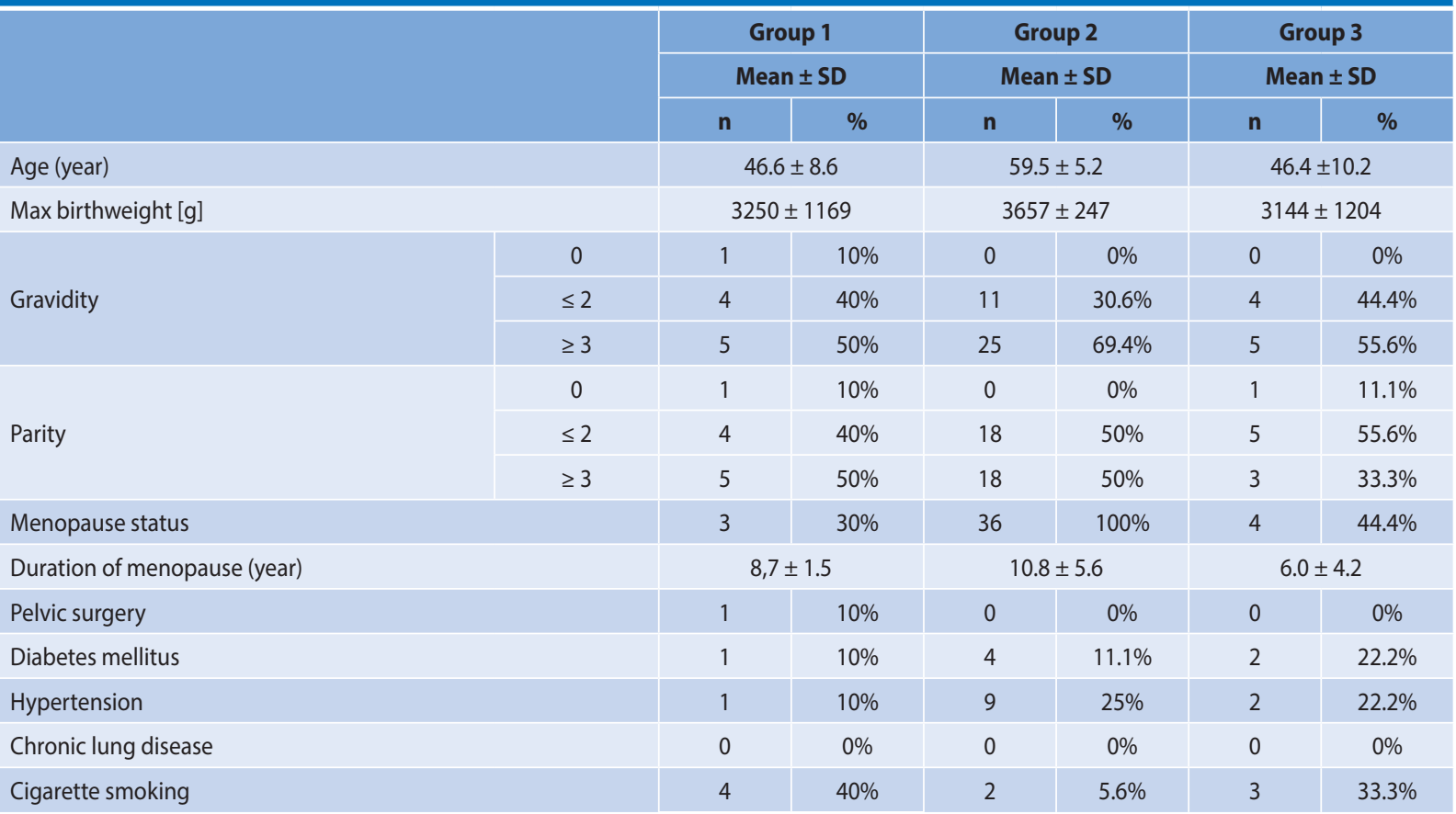

SD — standard deviation

Table 2. Radiotherapy techniques and doses

\begin{tabular}{|c|c|c|c|c|c|c|c|}
\hline & & \multirow{2}{*}{\multicolumn{2}{|c|}{$\begin{array}{c}\text { Group } 1 \\
\text { Mean } \pm \text { SD }\end{array}$}} & \multirow{2}{*}{\multicolumn{2}{|c|}{$\begin{array}{c}\text { Group } 2 \\
\text { Mean } \pm \text { SD }\end{array}$}} & \multirow{2}{*}{\multicolumn{2}{|c|}{$\begin{array}{c}\text { Group } 3 \\
\text { Mean } \pm \text { SD }\end{array}$}} \\
\hline & & & & & & & \\
\hline & & $\mathbf{n}$ & $\%$ & $n$ & $\%$ & $\mathbf{n}$ & $\%$ \\
\hline \multirow{3}{*}{ RT techniques } & BRT & 0 & $0 \%$ & 5 & $13.9 \%$ & 0 & $0 \%$ \\
\hline & BRT & 0 & $0 \%$ & 13 & $36.1 \%$ & 0 & $0 \%$ \\
\hline & $B R T+E R T$ & 10 & $100 \%$ & 18 & $50.0 \%$ & 9 & $100 \%$ \\
\hline \multicolumn{2}{|l|}{ ERT dose [cGy] } & \multicolumn{2}{|c|}{4500} & \multicolumn{2}{|c|}{$4570 \pm 184$} & \multicolumn{2}{|c|}{$4940 \pm 611$} \\
\hline \multicolumn{2}{|l|}{ BRT dose [cGy] } & \multicolumn{2}{|c|}{$1680 \pm 278$} & \multicolumn{2}{|c|}{$1609 \pm 464$} & \multicolumn{2}{|c|}{$2153 \pm 594$} \\
\hline \multicolumn{2}{|l|}{ Total dose [cGy] } & \multicolumn{2}{|c|}{$6180 \pm 278$} & \multicolumn{2}{|c|}{$4963 \pm 1357$} & \multicolumn{2}{|c|}{$7093 \pm 985$} \\
\hline
\end{tabular}

SD — standard deviation, BRT — brachytherapy, ERT — external radiotherapy 
Table 3. Pre and post-treatment incontinence occurence and results of Q-type test

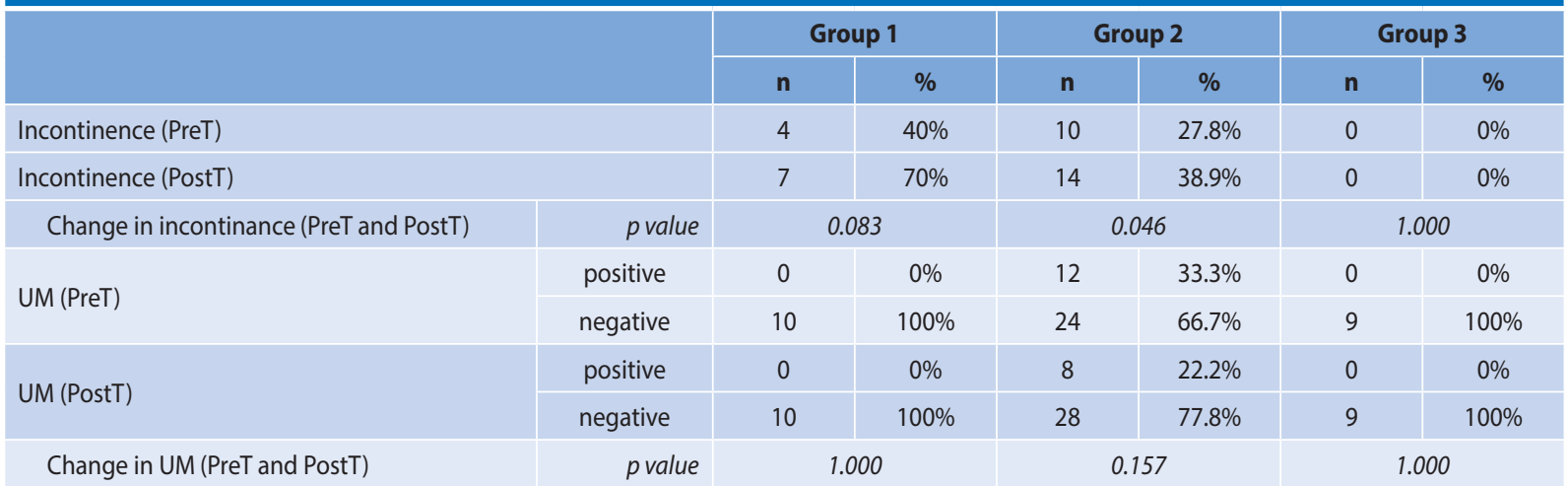

UM — urethral mobility, PreT — pre-treatment, PostT — post-treatment

\begin{tabular}{|c|c|c|c|c|}
\hline & & Group 1 & Group 2 & Group 3 \\
\hline FUUV [ml] (PreT) & & $335.9 \pm 85.2$ & $195 \pm 80.1$ & $202.9 \pm 55.3$ \\
\hline FUUV [ml] (PostT) & & $258.1 \pm 133.0$ & $186.9 \pm 89.1$ & $220.9 \pm 39.0$ \\
\hline Change in FUUV (PreT and PostT) & pvalue & 0.114 & 0.649 & 0.593 \\
\hline NUUV [ml] (PreT) & & $460.8 \pm 109.3$ & $351.2 \pm 119.0$ & $326.7 \pm 87.8$ \\
\hline NUUV [ml] (PostT) & & $387.3 \pm 116.1$ & $301.8 \pm 101.7$ & $280.6 \pm 45.1$ \\
\hline Change in NUUV (PreT and PostT) & $p$ value & 0.093 & 0.037 & 0.440 \\
\hline SUUV [ml] (PreT) & & $570.5 \pm 96.0$ & $485.3 \pm 145.3$ & $466.2 \pm 79.5$ \\
\hline SUUV [ml] (PostT) & & $494.6 \pm 144.0$ & $393.8 \pm 122.8$ & $366.6 \pm 85.7$ \\
\hline Change in SUUV (PreT and PostT) & p value & 0.114 & 0.000 & 0.138 \\
\hline
\end{tabular}

Data are presented as mean \pm SD. FUUV — first urge tu urinate volume, PreT — pre-treatment, NUUV — normal urge to urinate volume, PostT — post-treatment, SUUV — severe urge to urinate volume

Table 5. Changes in bladder capacity, amount of residual urine, maximal vesical pressure and detrusor pressure

\begin{tabular}{|c|c|c|c|c|}
\hline & & Group 1 & Group 2 & Group 3 \\
\hline Capacity [ml] (PreT) & & $662.2 \pm 82.7$ & $600.2 \pm 124.8$ & $593.0 \pm 69.1$ \\
\hline Capacity [ml] (PostT) & & $593.0 \pm 68.9$ & $490.0 \pm 92.6$ & $510.0 \pm 103.3$ \\
\hline Change in capacity (PreT and PostT) & $p$ value & 0.005 & 0.000 & 0.05 \\
\hline Residual urine [ml] (PreT) & & $5.1 \pm 1.2$ & $4.0 \pm 1.3$ & $4.4 \pm 0.5$ \\
\hline Residual urine [ml] (PostT) & & $4.8 \pm 0.8$ & $4.1 \pm 1.0$ & $4.4 \pm 0.5$ \\
\hline Change in residual urine (PreT and PostT) & $p$ value & 0.180 & 0.914 & 0.257 \\
\hline $\operatorname{MVP}\left[\mathrm{cm} / \mathrm{H}_{2} \mathrm{O}\right](\mathrm{PreT})$ & & $136.0 \pm 24.4$ & $129.3 \pm 40.1$ & $132.2 \pm 50.1$ \\
\hline MVP [cm/ $\left.\mathrm{H}_{2} \mathrm{O}\right]$ (PostT) & & $100.2 \pm 32.6$ & $130.2 \pm 56.9$ & $89.4 \pm 30.8$ \\
\hline Change in MVP (PreT and PostT) & $p$ value & 0.012 & 0.793 & 0.008 \\
\hline $\mathrm{MDP}\left[\mathrm{cm} / \mathrm{H}_{2} \mathrm{O}\right](\mathrm{PreT})$ & & $89.9 \pm 31.4$ & $69.4 \pm 23.8$ & $66.8 \pm 43.8$ \\
\hline MDP $\left[\mathrm{cm} / \mathrm{H}_{2} \mathrm{O}\right]$ (PostT) & & $58.7 \pm 23.9$ & $77.7 \pm 41.6$ & $61.7 \pm 41.7$ \\
\hline Change in MDP (PreT and PostT) & pvalue & 0.009 & 0.338 & 0.515 \\
\hline
\end{tabular}

Data are presented as mean \pm SD. PreT — pre-treatment, MVP — maximmal vesical pressure, PostT — post-treatment, MDP — maximal detrusor pressure 
decreases were observed in the capacity after treatment in Groups 1 and 2 ( $p=0.005$ and $p<0.001$, respectively). However, no significant differences in residual urine volumes before and after treatment were observed in any of the three groups (Table 5).

\section{DISCUSSION}

UI and urinary system disorders impair patient QoL and may lead to serious psychological, social, and sexual problems in women. Surgery and RT in gynecologic cancers are among the factors affecting the prevalence of UI [10]. Additionally, advanced age and comorbidities, which are common in these patients, may also adversely affect continence. In our study, mean patient age was $46 \pm 8.6,59.5 \pm 5.2$, and $46.4 \pm 10.2$ years for Groups 1, 2 and 3, respectively. Numerous authors have emphasized age as the main factor promoting higher both, "urge" and "stress" incontinence. Also, hysterectomies performed in old age intensify their effects on the bladder function [10-12].

Short-term effects of hysterectomy and its influence on the lower urinary system functioning have been reported in retrospective studies [11,13-16]. Frequency and urgency have been determined as the most common symptoms. It has also been reported that these symptoms usually disappear approximately in three to six months [17]. Prospective studies on the short-term effects of hysterectomy confirmed lack of any detrimental effect of hysterectomy on bladder function [12, 18-20]. In a study by El-Youkhy et al., an urodynamic assessment of hysterectomized patients six months after surgery revealed no detrimental effects and resolution of urinary dysfunction symptoms was observed [20]. As far as studies on long-term effects of hysterectomy are concerned, to the best of our knowledge, a three-year observational study by Gustafsson et al., has been the only long-term prospective study in the literature [21]. However, no urodynamic assessment was performed in that study as only patient symptoms were investigated and no significant differences were found for the frequency of urge incontinence, stress incontinence, or voiding patterns [21]. As for the available literature, only a few studies address the factors affecting incontinence, in terms of the number of patients, length and methods of monitoring, etc. In order to have a final result on individual effects of simple hysterectomy plus adjuvant RT, larger prospective and randomized studies with long-term monitoring should be designed.

Also, we found no statistically significant differences in post-treatment frequency of incontinence and urethral mobility results in RT after simple hysterectomy group. Similarly, we also found no significant differences in the urodynamic parameters six months after treatment. Based on these results and the available literature, simple hysterectomy fol- lowed by adjuvant RT does not seem to cause lower urinary system dysfunction.

Lower urinary system dysfunction is a well-known and frequent (reaching $80 \%$ in some series) complication of radical hysterectomy. Since the rate of developing a fistula has decreased below $1 \%$, UI has become the most common surgery-related morbidity among $\mathrm{RH}$ patients. If autonomic nerve injury occurs during $\mathrm{RH}$, urinary dysfunction, sexual dysfunction, and colorectal motility ailments may emerge according to the degree of the injury. The most common disorders include loss of sensation, hypertonic bladder, or hypo/a-contractile bladder, urgency and stress incontinence [22]. Axelsen et al., investigated 396 patients who filled out a questionnaire regarding urinary symptoms after $\mathrm{RH}$. Over one-third developed UI and urine retention, however 78\% of them did not consider these symptoms to constitute a problem [23]. Villena-Heinsen et al., performed a preand postoperative urodynamic assessment of 33 patients who underwent $\mathrm{RH}$. While they observed no changes in urethral resistance, functional length, or urethral pressure, they found the maximal bladder capacity to decrease from $615 \mathrm{~mL}$ to $503 \mathrm{~mL}$ in these patients. They also found a temporary decrease in maximal urinary flow and detrusor pressure and a delay in the first urge to urinate [24]. These authors pointed that incontinence was a common occurrence after $\mathrm{RH}$. The condition regressed spontaneously in a considerable number of patients, however a urodynamic investigation had to be performed in cases of incontinence which persisted for over a year. In our study, we performed urodynamic tests six months after RT following $\mathrm{RH}$ and found a statistically significant, but clinically nonsignificant, decrease in bladder capacity (from $662 \mathrm{~mL}$ to $593 \mathrm{~mL}$ ). Since normal bladder capacity is between $350-400 \mathrm{~mL}$ in healthy patients, this decrease may be of little significance in normal life. Additionally, we also found a statistically significant decrease in the maximal vesical and detrusor pressures. Although there was a decrease in the first urge to urinate, normal urge to urinate, and severe urge to urinate volumes in urodynamic terms, the decrease was statistically insignificant. Postoperatively, $70 \%$ of the patients complained of stress incontinence, while only $40 \%$ of them reported this complaint preoperatively. Also, there was an increase in the rates of frequent urination and urgency incontinence postoperatively. Considering that our findings, which are consistent with the literature, are early-stage results, we can expect that there will be a subsequent regress in urinary dysfunction symptoms over a long-term period of time.

Farquharson et al., studied early-stage effects of RT on bladder and urethral functions. They compared $30 \mathrm{pa}-$ tients who underwent only RH due to their cervical cancer, 30 patients who received only $\mathrm{RT}$, and 30 patients treated with $\mathrm{RH}$ and adjuvant $\mathrm{RT}$ with respect to urodynamics and 
symptoms. Voiding disorders and loss of urinary sensation were more often observed in the $\mathrm{RH}$ group and the $\mathrm{RH}$ plus adjuvant RT group. While $50 \%$ of the $\mathrm{RH}$ patients developed a strained urination pattern, this problem developed in $10 \%$ of the patients from the RT group. The only difference between the $\mathrm{RH}$ group and the $\mathrm{RH}$ plus adjuvant $\mathrm{RT}$ group was about bladder compliance but the difference was insignificant as well. Six months after treatment the incontinence rates were $23 \%$ in the RT group, $26 \%$ in the $\mathrm{RH}$ group, and $63 \%$ in the RH plus adjuvant RT group. The emergence of lower urinary system symptoms after RT was particularly linked to a decrease in compliance [25]. In another study by Lin et al., where primary RT and RT following RH were compared, strained urination and low compliance were found in both groups, but a significant decrease in bladder capacity was found in the RT following RH group [26]. In our study, we compared the six-month data of RH plus adjuvant RT and primary RT patients. We found a greater decrease in the first urge to urinate, normal urge to urinate, and severe urge to urinate volumes in the RH plus adjuvant RT group, while the decrease in bladder capacity was greater in the primary RT group. Also, a decrease in maximal vesical pressure and maximal detrusor pressure was greater in the $\mathrm{RH}$ plus adjuvant RT group.

We claimed that pelvic RT might caused demyelination on pelvic nerves and fibrosis on the bladder wall. Behr et al., reported that when the radiofibrosis associated with urge incontinence occurred, decreased bladder capacity and increased bladder tonus could be found concurrently, but these symptoms were partially recoverable [27]. Parkin et al., urodynamically assessed primary RT patients due to cervical cancer and found a significant decrease in the first urge to urinate volumes and maximal cystometric capacities. They claimed that urgency, frequency, and urge incontinence caused by decreased bladder capacity alone could not be the only reason, and that detrusor instability also played a role in the occurrence of these symptoms [4]. Barraclough et al., in their large-scale prospective study with 226 patients, observed that urgency, frequency and UI occurred in the early period following RT in these patients, but a significant increase occurred in the third year, especially in urgency and frequency [28]. In our study, the pre-RT urodynamic assessments of the primary RT patients was normal and a decrease was observed in the normal urge to urinate, severe urge to urinate, and maximal bladder capacity at 6-month follow-up. While there was a decrease in the maximal vesical pressure, there was no significant difference in maximal detrusor pressure. We also observed that the urgency-frequency symptoms were clinically more obvious in the primary RT group after six months. There were no complaints of stress incontinence in any of the adjuvant RT after hysterectomy patients, either pre-treatment or six months after treat- ment. Average bladder capacity was markedly lower after six months in the primary RT group than in the adjuvant RT following hysterectomy group. We related this result due to dose difference of approximately 1000 cGy on average between the adjuvant and primary RT protocols (6180 cGy vs. 7093 cGy, respectively).

Our study is not without limitations. First of all, the sample size was relatively small and we did not evaluate the effect of radical surgery alone, which might have helped us to assess the impact of radiation separately on urinary system functions. However, the findings suggest that adjuvant RT following $\mathrm{RH}$ may result in the lower urinary system dysfunction, although this might confound the results for patients in the radiation cohort who did not receive uniform radiation treatment. Although our study consisted of combined analysis of objective and subjective data obtained from a limited number of patients, we believe that it will serve as a guide for other prospective studies with larger sample size and longer monitoring periods. As cancer treatments aim at prolonging the survival without impairing patient QoL, further studies will be necessary to determine how to prevent urinary system dysfunction or decrease the detrimental effects during treatment.

\section{Conflict of interest}

The authors declare that there is no conflict of interest.

\section{REFERENCES}

1. Cardozo L, Staskin D. Textbook of Female Urology and Urogynecology. $2^{\text {nd }}$ ed. Informa Healthcare 2006, 115-141.

2. Chen GD, Lin LY, Wang PH, Lee HS. Urinary tract dysfunction after radical hysterectomy for cervical cancer. Gynecol Oncol. 2002, 85, 292-297.

3. Minini GF, Simeone C, Zambolin T, [et al.]. Longterm functional sequelae of the urinary tract following radical surgical treatment of carcinoma of the cervix with and without extended radiation therapy. Int Urogynecol J. 1992, 3, 8-11.

4. Parkin DE, Davis JA, Symonds RP. Long-term bladder symptomatology following radiotherapy for cervical carcinoma. Radiother Oncol. 1987, 9, 195-199.

5. Walters MD, Karam MM. Urogynecology and Reconstructive Pelvic Surgery. $3^{\text {rd }}$ ed. Mosby Elsevier 2007, 2, 17-28, 425-435.

6. Corcos J, Beaulieu S, Donovan JL, [et al.]. The symptom and quality of life committee of the first international consultation on incontinence. Quality of life assessment in men and women with urinary incontinence. J Urol. 2002, 168, 896-905.

7. Ory MG, Wyman JF, Yu L. Psychosocial factors in urinary incontinence. Clin Geriatr Med 1986, 2, 657-671.

8. Hsu TH, Rackley RR, Appell RA. The supine stress test: a simple method to detect intrinsic urethral sphincter dysfunction. JUrol. 1999, 162, 460-463.

9. Swift SE, Yoon EA. Test-retest reliability of the cough stress test in the evaluation of urinary incontinence. Obstet Gynecol. 1999, 94, 99-102.

10. Brown JS, Sawaya G, Thom DH, [et al.]. Hysterectomy and urinary incontinence: a systematic review. Lancet. 2000, 356, 535-539.

11. van der Vaart $\mathrm{CH}$, van der Bom JG, de Leeuw JR, [et al.]. The contribution of hysterectomy to the occurrence of urge and stress urinary incontinence symptoms. BJOG. 2002, 109, 149-154.

12. Kjerulff $H$, Langenberg $P W$, Greenaway $L$, [et al.]. Urinary incontinence and hysterectomy in a large prospective cohort study in American women. J Urol. 2002, 67, 2088-2092.

13. Milsom I, Ekelund $P$, Molander $U$, [et al.]. The influence of age, parity, oral contraception, hysterectomy and menopause on the prevalence of urinary incontinence in women. J Urol. 1993, 149, 1459-1462. 
14. Harris WJ. Complications of hysterectomy. Clin Obstet Gynecol. 1997, 40, 928-938.

15. Gunes G, Gunes A, Pehlivan E. Malatya Yesilyurt Saglık Ocagı bölgesindeki eriksin kadınlarda üriner inkontinans prevalansı ve etkili faktörler (Prevalance of uriner incontinans of women living in area of Malatya Yeşilyurt health care center and effective factors). Turgut Özal Tıp Dergisi. 2000, 7, 54-57.

16. Neumann G, Olesen PG, Hansen V, [et al.]. The short-term prevalence of de novo urinary symptoms after different modes of hysterectomy. Int Urogynecol J. 2004, 15, 1419-1423.

17. Griffith-Jones MD, Tufnell D. Urinary symptoms after total abdominal hysterectomy - a review. Int Urogynecol J. 1994, 5, 61-63.

18. Carlson KJ. Outcomes of hysterectomy. Clin Obstet Gynecol. 1997, 40, 939-946.

19. Virtanen $\mathrm{H}$, Makinen J, Tenho T, [et al.]. Effects of abdominal hysterectomy on urinary and sexual symptoms. Br J Urol. 1993, 72, 868-872.

20. El-Toukhy A, Hefni M, Davies A, [et al.]. The effect of different types of hysterectomy on urinary and sexual functions: a prospective study. J Obstet Gynecol. 2004, 24, 420-425.

21. Gustafsson C, Ekström S, Brismar D, [et al.]. Urinary incontinence after hysterectomy; three-year observational study. Urology. 2002, 68, 769-774.
22. Benedetti-Panici P, Zullo MA, Plotti F, [et al.]. Long-term bladder function in patients with locally advanced cervical carcinoma treated with neoadjuvant chemotherapy and type 3-4 radical hysterectomy. Cancer. 2004, 100, 10-16.

23. Axelsen SM, Petersen LK. Urogynaecological dysfunction after radical hysterectomy. Eur J Surg Oncol. 2006, 32, 445-449.

24. Villena-Heinsen C, Metzgeroth GS, Tossounidis I, [et al.]. Urodynamic parameters and continence after radical Wertheim-Meigs-Okabayashi hysterectomy. Zentralbl Gynakol. 1997, 119, 476-482.

25. Farquharson DIM, Shingleton HM, Orr JW, [et al.]. The short-term effect of radical hysterectomy on urethral and bladder function. Br J Obstet Gynaecol. 1987, 94, 351-357.

26. Lin $\mathrm{HH}$, Sheu $\mathrm{BC}$, Lo $\mathrm{MC}$, [et al.]. Abnormal urodynamic findings after radical hysterectomy or pelvic irradiation for cervical cancer. Int J Gynecol Obstet. 1998, 63, 169-174.

27. Behr J, Winkler M, Willgeroth F. Functional changes in the lower urinary tract after irradiation of cervix carcinoma. Strahlenther Onkol. 1990, 166, 135-139.

28. Barraclough LH, Routledge JA, Farnell DJ, [et al.]. Prospective analysis of patient-reported late toxicity following pelvic radiotherapy for gynaecological cancer. Radiother Oncol. 2012, 10, 327-332. 